\title{
Diagnostik und Therapie des Melanoms im Wandel der letzten 25 Jahre Standortbestimmung und Ausblick
}

\author{
Changes of the Diagnostic and Therapeutic Standards for Malignant Melanoma in the Last 25 Years \\ State of the Art and Upcoming Advances
}

Autoren

Institute

Korrespondenzadresse

Priv.-Doz. Dr. med. Hans Starz

Labor für Dermatohistologie

und Oralpathologie München

Bayerstr. 69

80335 München

hstarz@web.de

\author{
H. Starz ${ }^{1,2}$, B.-R. Balda ${ }^{1}$, C. Haas ${ }^{3}$, K.-U. Krämer ${ }^{1}$, C. Pfeiffer ${ }^{1}$, J. Welzel ${ }^{1}$ \\ ${ }^{1}$ Klinik für Dermatologie und Allergologie, Klinikum Augsburg \\ ${ }^{2}$ Labor für Dermatohistologie und Oralpathologie München \\ ${ }^{3}$ Institut für Pathologie, Klinikum Augsburg
}

\section{Zusammenfassung \\ $\nabla$}

Im zurückliegenden Vierteljahrhundert hat sich unser Verständnis maligner Melanome enorm erweitert, und dies führte zu einer Vielzahl neuer Diagnostik- und Behandlungsstrategien.

Nach einer als erfolgreich bewerteten Pilotphase in Schleswig-Holstein wurde wegen der hohen und ständig noch steigenden Hautkrebsinzidenz 2008 die Hautkrebsfrüherkennung auf Kosten der gesetzlichen Krankenkassen deutschlandweit eingeführt.

Die Früherkennung und -beseitigung regionärer Lymphknotenmetastasen von Patienten mit mitteldicken und dicken Hautmelanomen gelang durch die Etablierung und innerhalb weniger Jahre erreichten Optimierung der Sentinel-Lymphonodektomie. Dabei erweist sich zudem die mikromorphometrische Klassifikation des SentinelLymphknotenbefalls als erstrangiges Prognoseund Therapieleitkriterium, etwa für die Indikation zur radikalen Komplettierungsdissektion oder zur adjuvanten Interferon-alpha-Therapie. In fortgeschrittenen Metastasierungsstadien stehen erstmals erfolgreichere Systemtherapien als die Dacarbazin-Monotherapie zur Verfügung. Bei knapp 50\% der Melanome findet sich in der BRAF-Kinase des MAPK-Signaltransduktionsweges eine aktivierende V600-Mutation, die sich

\section{Einleitung}

$\nabla$

In der Dermatologie ist das maligne Melanom ein Paradebeispiel sowohl für einen geradezu exponentiell zunehmenden Wissenszuwachs als auch für den dadurch bedingten Wandel im Krankheitsverständnis und der Behandlungsoptionen in den vergangenen 25 Jahren. Mit Fug und Recht darf behauptet werden, dass einige Aspekte dieser für Melanompatienten positiven Entwicklung maßgeblich im Klinikum Augsburg vorangetrie- wirksam mit den oral verfügbaren Medikamenten Vemurafenib oder Dabrafenib blockieren lässt. Die so rasch erzielbare Metastasenregression wird durch Kombination mit einem MEKInhibitor (z.B. Trametinib) noch nachhaltiger. Von den Immuntherapien erreichten Antikörper gegen die immunbremsenden T-Zell-Rezeptoren CTLA4 und PD-1 die besten Therapieergebnisse. Obwohl es darunter selten zu Vollremissionen kommt, können bei einem Teil der Patienten in fortgeschrittenen Melanomstadien anhaltende Tumorkontrollen erzielt werden. Die höchste Tumorkontrollrate ergab sich in Studien mit der Kombination des PD-1-Antikörpers Nivolumab und des CTLA4-Antikörpers Ipilimumab.

Die sinnvolle Nutzung und Kombination dieser innovativen therapeutischen Eingriffe eröffnet erstmals die Chance, die Melanommortalität trotz weiter steigender Inzidenz zu senken. Schon jetzt zeichnet sich allerdings ab, dass dies nicht ohne präzise Charakterisierung von Melanomsubentitäten sowie die sorgfältige Herausarbeitung von Prognosekriterien gelingen wird. Eine derartig differenzierte Patientenversorgung lässt sich sowohl aus medizinischer als auch ökonomischer Sicht am besten durch interdisziplinäre Kooperation im Rahmen von Hautkrebszentren realisieren.

ben wurden [1 -9]. Wichtig ist dabei die interdisziplinäre Kooperation nicht nur im Hinblick auf den Erfahrungsaustausch, sondern auch bezüglich der unmittelbaren praktisch-klinischen Patientenversorgung.

Die Dringlichkeit von Therapiefortschritten ergab sich u.a. aus der rasanten Inzidenzzunahme des malignen Melanoms [10,11]. Drei der in den 90er-Jahren besonders diskutierten Fragen seien herausgegriffen: 
1. Welche Präventionsstrategien sind erfolgversprechend [12]?

2. Soll bei mitteldicken und dicken bzw. ulzerierten Hautmelanomen eine elektive Dissektion der klinisch und sonografisch unauffälligen regionären Lymphknoten unmittelbar mit der Primärtumorresektion verbunden werden oder ist es besser, sich stattdessen auf eine sorgfältige Nachbeobachtung der Lymphknotenregion zu beschränken und erst im Falle nachgewiesener Metastasen die betroffene Lymphknotenstation radikal auszuräumen $[13,14]$ ?

3. Mit welchen Systemtherapeutika kann Patienten in fortgeschrittenen, operativ nicht mehr R0-sanierbaren Stadien besser geholfen werden als mit der zwar als Standard akzeptierten, jedoch nie als eindeutig überzeugend effektiv dargestellten Dacarbazin-Monotherapie [15-17]?

\section{Früherkennungsstrategien}

Nachdem Primärpräventionsbemühungen weltweit die Inzidenzzunahme maligner Melanome nicht nennenswert hatten verhindern können, wurden Früherkennungsstrategien um so wichtiger, wobei insbesondere hochwertige Dermatoskopiegeräte dem Geübten zusätzliche technische Hilfen bei der Differenzierung zwischen Melanomen und benignen Pigmentzellveränderungen geben können [18]. Hervorzuheben ist die computergestützte sequenzielle Dermatoskopie, die insbesondere unmittelbare Bildvergleiche zu Voraufnahmen der jeweiligen Pigmentmale ermöglicht, und durch Mustererkennungsalgorithmen sogar Verdachtsmomente quantifizieren kann, ohne freilich das Erfahrungswissen des Untersuchers zu ersetzen. In Deutschland wurde 2008 nach einer Pilotphase in Schleswig-Holstein von 2003 bis 2004 für gesetzlich Versicherte ab 35 Jahren eine flächendeckende Hautkrebsvorsorgeuntersuchung zu Lasten der Krankenkassen im Rhythmus von zwei Jahren eingeführt [19, 20]. Schleswig-Holstein registrierte fünf Jahre nach Abschluss der Screeningperiode eine in Deutschland einmalige Senkung der Melanommortalität um mehr als $50 \%$, was zumindest teilweise auf diese Maßnahme zurückgeführt wurde [21]. Die Auswirkungen des bundesweiten Screenings werden derzeit evaluiert. In den letzten Jahren hat sich auch die Interpretation multipler dysplastischer Nävi gewandelt. Wurde früher davon ausgegangen, dass dysplastische Nävi ein hohes Risiko für die Entwicklung maligner Melanome beinhalteten, weshalb vielerorts ihre prophylaktische Exzision empfohlen wurde [22], gilt heute, dass sie lediglich einen unabhängigen prognostischen Marker für das Melanomrisiko darstellen. Das Hauptproblem ist vielmehr, bei diesen Patienten unter zahlreichen atypischen Pigmentmalen ein neu entwickeltes malignes Melanom möglichst früh zu erkennen [23]. Bei klinisch und dermatoskopisch unklaren Läsionen ermöglichen mittlerweile weitere nichtinvasive diagnostische Verfahren, unnötige Exzisionen benigner Nävi zu vermeiden, aber auch maligne Melanome zu identifizieren. Hier ist insbesondere die konfokale Lasermikroskopie zu nennen, mit der hochauflösend und nebenwirkungsfrei epidermale und oberflächlich-dermale Veränderungen in Pigmentmalen analysiert werden können [24,25].

\section{Sentinel-Lymphonodektomie (SLNE)}

\section{$\nabla$}

Als Königsweg in der Frage der frühen Lymphknotenchirurgie erwies sich die SLNE, also die selektive und morbiditätsarme Entnahme und Untersuchung der ersten Ziellymphknoten im vom Primärmelanom ausgehenden Lymphabfluss, wie sie bereits R. Virchow 1863 definiert hat [26]. Nur bei Befall dieser „Wächter-(engl. Sentinel-)Lymphknoten (SLN) wird als Zweitoperation die radikale Komplettierungsdissektion (CLND) der gesamten betroffenen Lymphknotenregion angeschlossen. Diese 1992 von der Arbeitsgruppe um D.L. Morton und A.J. Cochran publizierte Methode [27] setzte sich dank der hochsignifikanten Verbesserung der regionären Tumorkontrolle fast weltweit sehr schnell durch und wurde beispielgebend auch für die Ersttherapie zahlreicher anderer Malignome übernommen (z.B. Mamma-, Prostata- und Magenkarzinom [28-31]. Für Hautmalignome, auch solche nichtmelanozytären Ursprungs [32-35], konnten in den Folgejahren noch einige Methodenverbesserungen z.B. durch Einführung der präoperativen Lymphszintigrafie und der intraoperativen Gammasondennutzung etabliert werden, die im Rahmen einer Konsensuskonferenz der weltweit führenden Zentren in Augsburg als Standards definiert wurden („Augsburg Consensus“) [2]. Nicht sicher beweisen ließ sich in einer weltweit durchgeführten, prospektiven und randomisierten Studie (MSLT-I) der Gesamtüberlebensvorteil durch SLNE mit selektiv bei SLN-Befall ergänzter CLND für die Gesamtheit der Melanompatienten, bei denen die Indikation zu diesem abgestuften Procedere gestellt wurde [36,37]). Für die Minderheit mit SLN-Mikrometastasen zeichnete sich dagegen ein Überlebensvorteil hochsignifikant ab [37]. Nach unserer eigenen Erfahrung ist seit Einführung der gammasondengesteuerten SLNE auch für die Gesamtheit unserer Patienten im Vergleich zu den stadiengleichen Patienten der vorausgegangenen Jahre eine Überlebensverbesserung hochsignifikant eingetreten ( $\bullet$ Abb. 1 ) $[8,38]$, die sich auch auf das fernmetastasenfreie und das rezidivfreie Überleben zurückverfolgen lässt ( $\bullet$ Abb. 2 und $\bullet$ Abb. 3).

Ganz ohne Zweifel besteht eine erstrangige prognostische Bedeutung des SLN-Status $[8,39]$. Patienten mit Melanomen über $0,75 \mathrm{~mm}$ Tumordicke erreichen bei metastasenfreien SLN eine melanombezogene 10-Jahre-Überlebensrate von $89 \%$, bei SLNBefall dagegen nur von 70\%. Eine noch viel genauere Prognoseabschätzung gelingt durch mikromorphometrische Klassifikation des SLN-Befalls [3,4,8,40-44]. Geeignete Messparameter sind die Tumoreindringtiefe, bezogen auf den Innenrand der SLN-Kapsel (S-Klassifikation, Tab.1), und der maximale Durchmesser der größten SLN-Metastase (Rotterdam-Klassifikation, Tab.2). Jüngst definierte der Erstautor dieser Arbeit (H.S.) aus diesen Parametern eine kombinierte „Augsburg-Rotterdam-Klassifikation“ ( $\bullet$ Tab.3), die eine noch bessere Prognosedifferenzierung aufweist als jede der Quellklassifikationen $(\bullet$ Abb.4). Leider kam keine der genannten Klassifikationen als Stratifikationskriterium für die prospektiv-randomisierten Studien (MSLT-II und die DeCOG-Wächterlymphknoten-Studie) zum Einsatz, bei denen es um den Wert bzw. die Verzichtbarkeit der CLND bei SLN-Befall geht. Sie hätten sicherlich differenziertere Antworten auf die Frage erlaubt, welchen Melanompatienten mit SLN-Befall sinnvoll eine CLND erspart bleiben kann und welchen besser nicht $[36,45]$. Auf der Basis von retrospektiven Verlaufsdaten kann nach Meinung der meisten Experten bei Patienten der AR-Klasse 1 auf eine radikale Komplettierungsdissektion der Lymphknoten verzichtet werden, allerdings unter engmaschigen sonografischen Kontrollen [8,42-45]. 


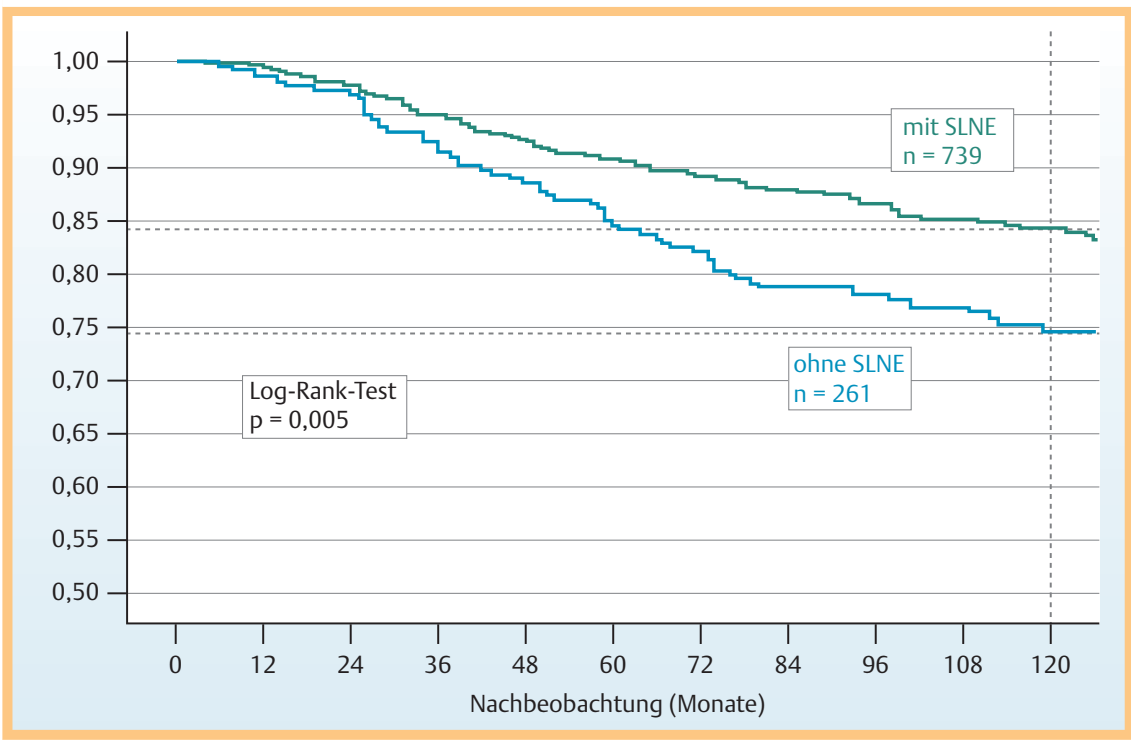

Abb. 1 Kaplan-Meier Kurven (mediane Nachbeobachtung 111 Monate) und Log-Rank-Test zum melanombezogenen Gesamtüberleben von 1000 Patienten des Klinikums Augsburg mit Melanomen $>0,75 \mathrm{~mm}$ Tumordicke ohne Metastasen im nicht-invasiven Erststaging. 261 dieser Patienten waren 1987-1993 ohne elektiven Lymphknoteneingriff erstversorgt worden, 739 in den Jahren 1995 - 2005 mit Gammasonden-gesteuerter Sentinel-Lymphonodektomie (SLNE) und je nach SLNBefund mit einer Komplettierungsdissektion der jeweiligen Lymphknotenregion. Die Vergleichbarkeit beider Patientengruppen zeigt sich $u$. a. an den fast deckungsgleichen Kurvenverläufen in den ersten Jahren der Nachbeobachtung.

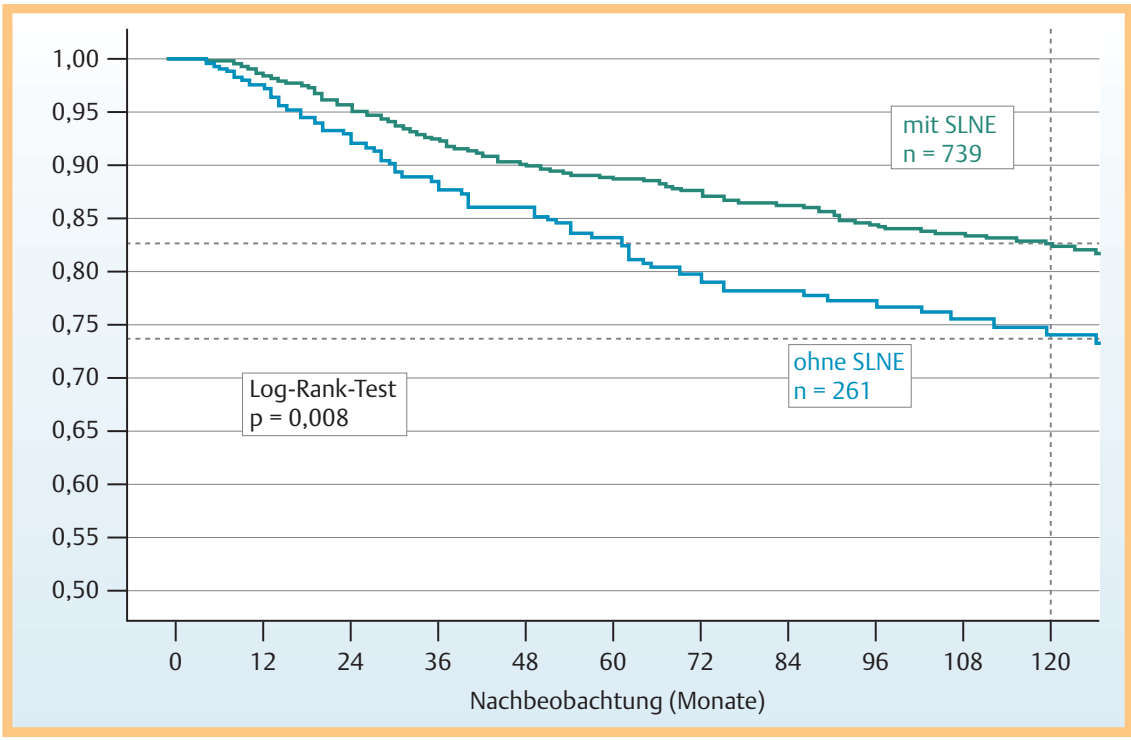

Abb.2 Kaplan-Meier Kurven und Log-Rank-Test zum fernmetastasenfreien Überleben der beiden Patientengruppen (s. $\odot$ Abb. 1).

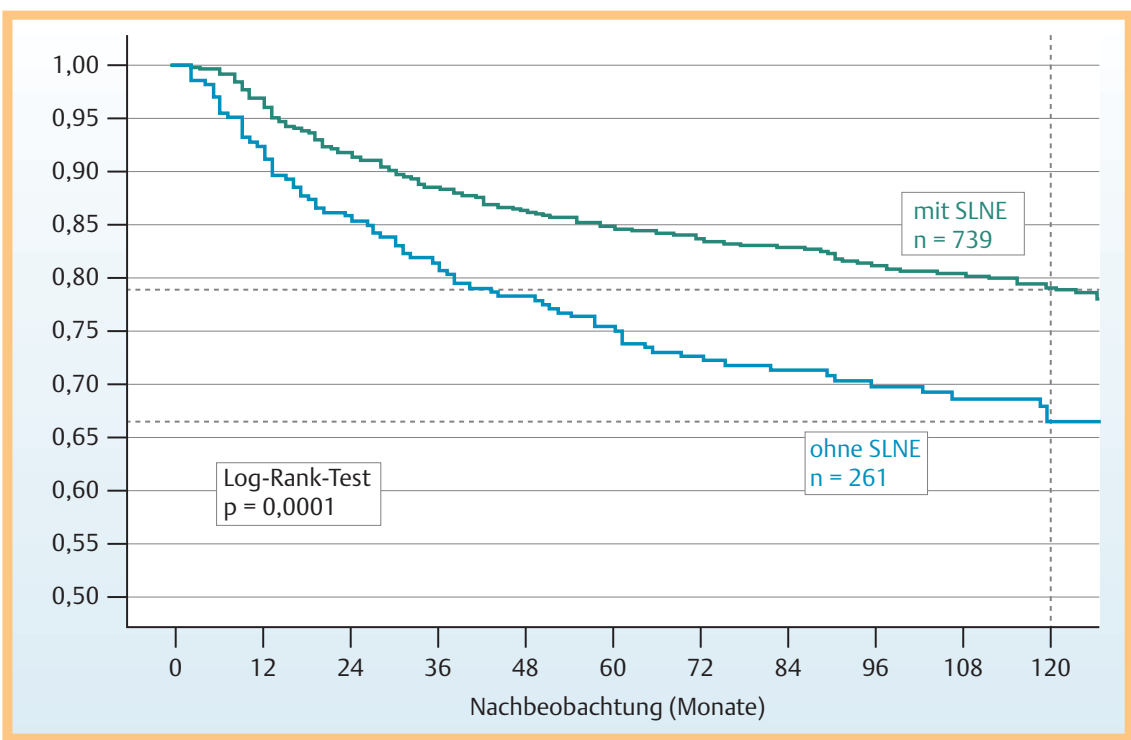

Abb.3 Kaplan-Meier Kurven und Log-Rank-Test zum rezidivfreien Überleben der beiden Patientengruppen (s.๑ Abb.1). 
Tab. 1 Definition der S-Klassifikation des Sentinel-Lymphknoten-(SLN-) Befalls beim malignen Melanom oder auch bei anderen Malignomen. Die Tumoreindringtiefe (TET) wird vom Innenrand der SLN-Kapsel bis zur tiefsten Tumorzelle innerhalb des SLN gemessen.

\begin{tabular}{|c|c|}
\hline \multicolumn{2}{|c|}{ S-Klassifikation } \\
\hline so & keine histologisch identifizierbare SLN-Metastase \\
\hline SI & Metastasen mit einer Tumoreindringtiefe $(T E T) \leq 0,3 \mathrm{~mm}$ \\
\hline SII & Metastasen mit $0,3 \mathrm{~mm}<\mathrm{TET} \leq 1 \mathrm{~mm}$ \\
\hline SIII & Metastasen mit TET > $1 \mathrm{~mm}$ \\
\hline
\end{tabular}

Tab.2 Definition der Rotterdam-Klassifikation des Sentinel-LymphknotenBefalls beim malignen Melanom. Der maximale Durchmesser ist als der größte Durchmesser des größten Melanomzellverbandes im SLN definiert.

\begin{tabular}{|l|l} 
Rotterdam-Klassifikation \\
\hline R0 & keine histologisch identifizierbare $S L N$-Metastase \\
\hline R1 & Metastasen mit maximalem Durchmesser $(M D) \leq 0,1 \mathrm{~mm}$ \\
\hline R2 & Metastasen mit $0,1 \mathrm{~mm}<M D \leq 1 \mathrm{~mm}$ \\
\hline R3 & Metastasen mit MD $>1 \mathrm{~mm}$
\end{tabular}

Tab.3 Definition der Augsburg-Rotterdam-(AR-)Klassifikation des SentinelLymphknoten-Befalls beim malignen Melanom.

\begin{tabular}{|ll|}
\hline \multicolumn{2}{|l|}{ Augsburg-Rotterdam-Klassifikation } \\
\hline AR0 & keine histologisch identifizierbare SLN-Metastase \\
\hline AR1 & $\begin{array}{l}\text { Metastasen mit maximalem Durchmesser }(\mathrm{MD}) \leq 0,1 \mathrm{~mm} \\
\text { und Tumoreindringtiefe }(\mathrm{TET}) \leq 0,3 \mathrm{~mm}\end{array}$ \\
\hline AR2 & $\begin{array}{l}\text { Metastasen mit jeglicher Messungskombination zwischen AR1 } \\
\text { und AR3 }\end{array}$ \\
\hline AR3 & Metastasen mit MD $>1 \mathrm{~mm}$ und TET $>1 \mathrm{~mm}$ \\
\hline
\end{tabular}

Dies relativiert auch das in den 1990er-Jahren von einigen Arbeitsgruppen verfochtene Ziel, die Sensitivität des Metastasennachweises im SLN mittels molekularer PCR-Nachweisverfahren weiter zu steigern $[46,47]$. Ohne histologische Gegenkontrolle kam es hierdurch zu hohen Raten falsch-positiver Befunde, die sich insbesondere auf die Existenz von Nävuszellen in SLN von etwa 30\% der Melanompatienten zurückführen ließen [7]. Füh- rend und diagnostisch maßgeblich sind auch heute noch die histologisch-immunhistochemischen Untersuchungsmethoden.

\section{Zielgerichtete Therapien mit Kinaseinhibitoren}

Medikamentöse Therapieversuche für das fortgeschritten metastasierte Melanom haben über mehrere Jahrzehnte enttäuscht, so dass selbst im Stadium IV oft heroische chirurgische Sanierungsbemühungen erfolgreicher waren als die Chemotherapie $[15,16$, 48].

Dies änderte sich entscheidend mit dem besseren Verständnis der Wachstumssteuerungsmechanismen und der hierfür wesentlichen Schlüsselmoleküle in den Tumorzellen. Die für das Melanom offenbar wichtigste Signalkaskade ist der sog. MAPKPathway ( $\triangle$ Abb.5). Bei fast 50\% der Melanome findet sich die BRAF-Kinase durch einen Aminosäureaustausch an der Position 600 so verändert, dass sie daueraktiviert ist und daher unkontrolliert Wachstumssignale weiterleitet [49-51]. Die häufigste zugrunde liegende „aktivierende“ Genmutation führt zum Ersatz von Valin durch Glutamat und wird als BRAF-V600E-Mutation bezeichnet. In Kopf-Hals-Melanomen ist die basische Aminosäure Lysin ein bevorzugter Austauschpartner von Valin (V600K). Selten ist Valin durch die ebenfalls basische Aminosäure Arginin substituiert (V600R). Gegen die so aktivierte BRAF-Kinase wurden blockierende Moleküle entwickelt, z.B. Vemurafenib und Dabrafenib, die heute führende Therapeutika für fortgeschrittene Melanome mit BRAF-V600-Mutationen darstellen [52 - 55]. Denn sie erwiesen sich als geeignet, in diesen Fällen regelhaft selbst weit fortgeschrittene Metastasierungsstadien innerhalb weniger Wochen in eine Teil- oder gar Vollremission zu bringen. Leider entwickeln sich meist aber innerhalb einiger Monate Therapieresistenzen mit der Folge erneuter Tumorprogression. Teilweise lassen sich diese in Analogie zur Antibiotikatherapie durch Kombination eines BRAF- mit einem MEK-Inhibitor, z.B. Trametinib, vermeiden oder hinauszögern [56,57]. Interessanterweise verhindert die Kombination meist sogar das Auftreten epithelialer Hauttumore, die bei alleiniger BRAF-Inhibitor-Therapie eine der häufigsten Nebenwirkungen darstellen. Gelöst ist das Problem der Resistenzen durch die bisher verfügbaren Kombinationen

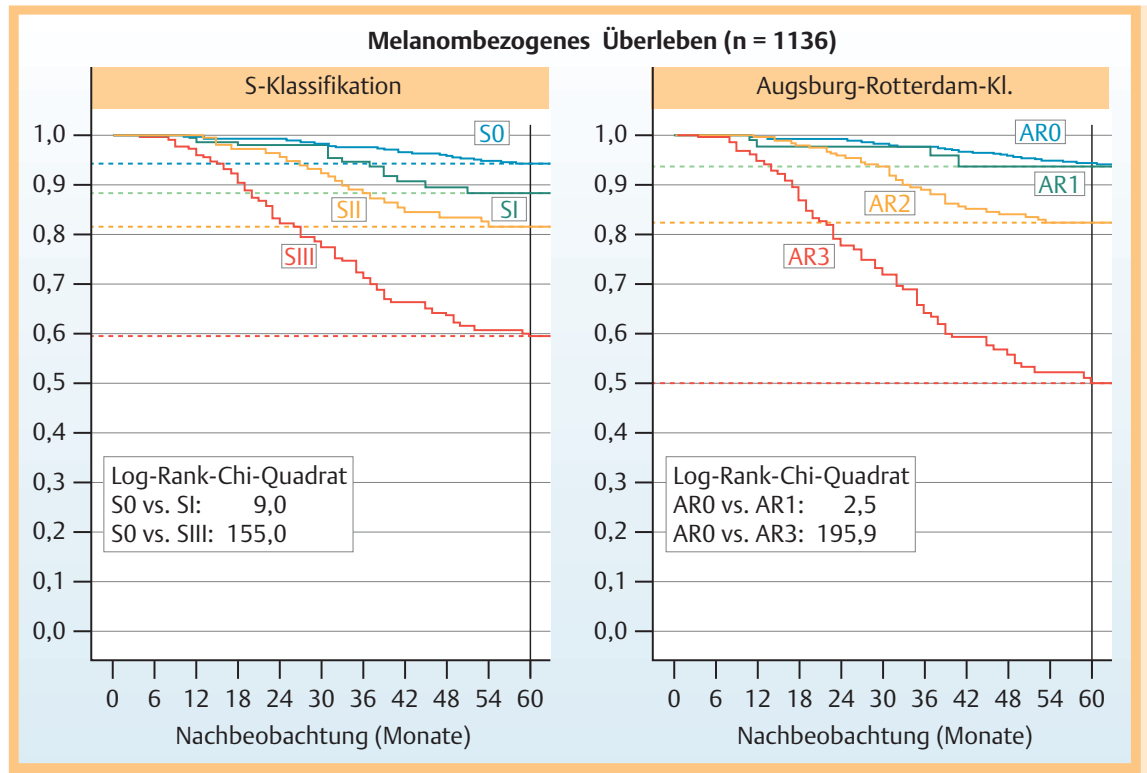

Abb.4 Gegenüberstellung der S- und der Augsburg-Rotterdam-(AR-)Klassifikation hinsichtlich ihrer prognostischen Aussagekraft für das melanombezogene Überleben. Beide erlauben bei den Patienten mit Melanommetastasen in SentinelLymphknoten (SLN) die Einteilung in eine Niedrig(SI, AR1), Mittel- (SII, AR2) und Hochrisikokategorie (SIII, AR3). Bei der AR-Klassifikation gelingt die Prognosedifferenzierung am besten: AR1-Patienten erreichen mit $93 \%$ eine gleich gute 5-Jahre-Überlebensrate wie AR0-Patienten, wohingegen $50 \%$ der AR3-Patienten in den ersten fünf Jahren nach Diagnose melanombedingt sterben. Die dargestellten Kaplan-Meier-Kurven geben die Erfahrungen aus zwei deutschen Hauttumorzentren wider (Klinikum Augsburg und Universitätsklinikum Göttingen). 


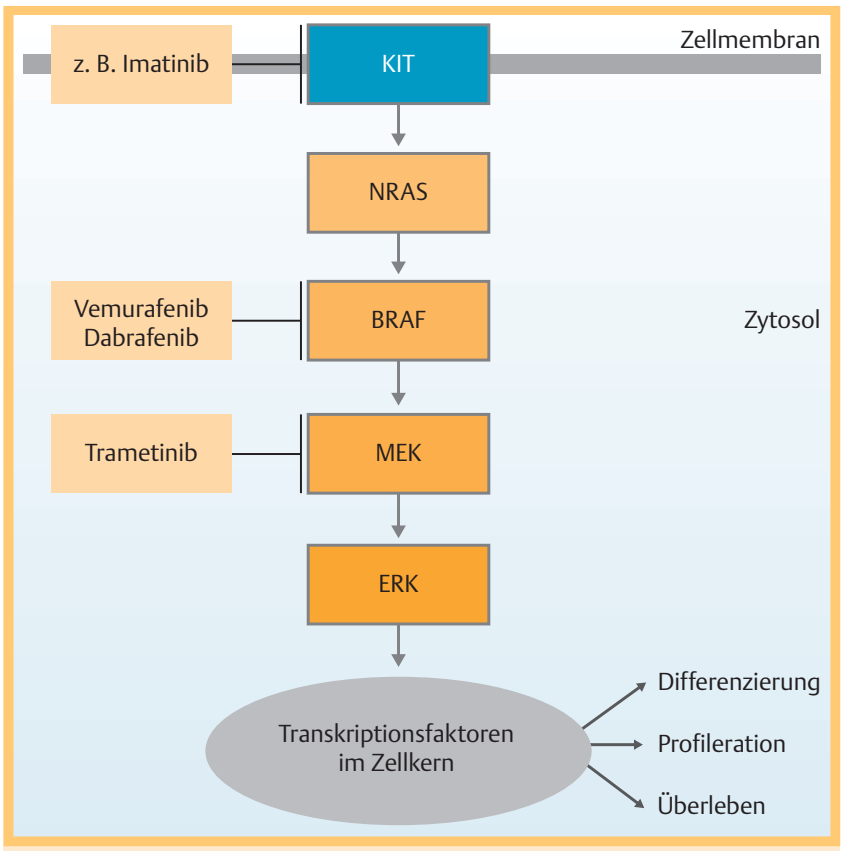

Abb.5 Schemadarstellung des MAPK-Signalwegs, beginnend mit der membranständigen Rezeptorkinase KIT über die zytosolischen Kinasen NRAS, BRAF, MEK und ERK bis zu den im Zellkern gelegenen Signalempfangsstellen. Rechts unten finden sich die Auswirkungen auf die Zelle. In Melanomzellen können KIT (zu<5\%), NRAS (zu ca. $20 \%$ ) oder BRAF (40$50 \%$ ) so alteriert sein, dass eine unkontrollierte Daueraktivierung resultiert. Links sind eine Reihe von Kinaseinhibitoren aufgeführt, die gezielt dagegen therapeutisch eingesetzt werden können.

von Kinaseinhibitoren aber keineswegs. Zudem bestehen für Melanome mit anderen Mutationen nur eingeschränkte therapeutische Erfahrungen mit Kinaseinhibitoren $[56,58]$.

\section{Immuntherapien \\ $\nabla$}

Kutane Melanome gehen nicht selten unter entzündlichen Begleiterscheinungen in partielle, gelegentlich sogar in komplette Spontanregression. Schon früh fokussierten sich therapeutische Bemühungen daher auf die Stimulation der Immunabwehr. Während Vakzinierungsansätze vielfach eher Immuntoleranz als Immunstimulation bewirkten und sich jedenfalls bisher in der Standardtherapie nicht durchsetzen konnten [59,60], wurden bessere Erfolge mit Cytokinen, insbesondere Interferon-alpha, und mit der Antikörperblockade derjenigen Rezeptoren auf T-Zellen erzielt, die die immunologische Aktivität normalerweise herunterregulieren [61-68].

Interferon-alpha wird in unterschiedlichen Dosierungen und chemischen Modifikationen (z. B. pegyliert) adjuvant bei Risikomelanompatienten z.B. nach Entfernung eines mikrobefallenen SLN empfohlen [61 -63]. Nach unserem Verständnis der Datenlage konnte eine therapeutische Überlegenheit hoher gegenüber den wesentlich besser vertragenen niedrigen Dosen nicht eindeutig erwiesen werden [64], und selbst in Niedrigdosisprotokollen (z.B. $3 \times 3$ Mio IE/Woche subkutan) muss das Risikoprofil der Medikation in jedem Einzelfall kritisch gegen die anzunehmende eher geringfügige Verminderung des Melanomrezidivrisikos abgewogen werden. In fortgeschritteneren Melanomstadien war Interferon selbst in Therapiekombinationen nicht signifikant erfolgreich [63] .
Ein Fortschritt gelang erst mit Antikörpern gegen den CTLA4-Rezeptor auf T-Zellen, von denen Ipilimumab nach erwiesener Überlebensverbesserung gegenüber der Standard-Dacarbazinmonotherapie die Erstlinientherapiezulassung 2013 in Deutschland erhielt [9,65-67]. Durch diese Bremsblockade im Immunsystem kann bei $22 \%$ der Patienten in fortgeschritteneren Melanomstadien eine anhaltende Tumorkontrolle auf meist niedrigem Tumorlastniveau erreicht werden [67]. Rasche Vollremissionen sind allerdings unter Ipilimumab kaum zu erwarten. Auch muss auf potenziell lebensbedrohende immunvermittelte Therapienebenwirkungen v. a. im Colon, an der Leber und im Endokrinum geachtet und ggf. rasch reagiert werden.

Noch besser ist das Nutzen-Risiko-Profil offenbar bei den jüngst zur Therapie inoperabel metastasierter Melanome zugelassenen PD1-Antikörpern Pembrolizumab und Nivolumab. Hier ergaben Studien eine anhaltende Tumorkontrolle bei etwa $40 \%$ der Patienten, und dies sogar bei rascherem Ansprechen und weniger problematischem Nebenwirkungsprofil [68]. Eine noch bessere Effizienz konnte jüngst in Studien durch Kombination von Nivolumab mit Ipilimumab erzielt werden [69-71]. Auch zeichnen sich erste Erfolge bei der Ermittlung von Biomarkern ab, die künftig vielleicht eine bessere Patientenselektion für die individuell erfolgversprechendste Therapie ermöglichen [72].

\section{Interdisziplinäre Kooperation und Zentrenbildung $\nabla$}

Für das maligne Melanom existiert seit 2013 eine S3-Leitlinie, die interdisziplinär in zahlreichen Arbeitsgruppen erarbeitet worden war [63]. Freilich führt die rasche Dynamik des Therapiefortschritts dazu, dass viele der Leitlinien kurz nach ihrer Publikation schon wieder einer Aktualisierung bedürfen. Wichtiger ist es daher, dass in multidisziplinären Tumorkonferenzen aktuelle Therapiekonzepte individuell für jeden Melanompatienten in einem kritischen Tumorstadium entwickelt, konsentiert und einer zügigen Umsetzung zugeführt werden. Im europäischen Vergleich ist die Versorgungssituation von Melanompatienten in Deutschland als gut zu bezeichnen, was sich in einer im Vergleich niedrigeren Tumordicke und Mortalität widerspiegelt [73]. Dies ist insbesondere darauf zurückzuführen, dass die Patienten einen schnellen Zugang zu Dermatologen haben und Diagnostik, operative und onkologische Therapie in einer Hand liegen.

\section{Ein Blick in die Zukunft}

$\nabla$

Das technische Instrumentarium zur präinvasiven Melanomfrüherkennung erweitert sich derzeit erheblich. Zu Grunde liegen besondere optische Verfahren wie die dynamische optische Kohärenztomografie oder die konfokale Lasermikroskopie, aber auch andere physikalische Methoden wie etwa die Multispektralanalyse und die Impedanzspektroskopie an Pigmentmalen $[25,26,74-76]$. Allerdings darf in Zweifelsfällen nie vergessen werden, dass der Goldstandard zur frühen Diagnosesicherung die histologisch-immunhistochemische Klärung nach Exzisionsbiopsie ist. Natürlich bleibt manchmal auch hierbei eine „Grauzone“ ungeklärter Dignität, in die molekularpathologische und genomische Untersuchungen mehr Licht bringen werden [72, 77]. Auch haben wir bereits begonnen zu lernen, mit Hilfe molekularer Charakterisierung prognostisch distinkte Melanomsubentitäten zu unterscheiden, nicht zuletzt auch im Hinblick auf ein sich immer weiter diversifizierendes Spektrum an Melanom- 
therapien und Therapiekombinationen [49-51]. Vermutlich werden neue Systemtherapien zunehmend auch adjuvant zur Verfügung stehen [78]. Das kann Patienten nach operativer Ersttherapie von Risikomelanomen möglicherweise eine deutliche Prognoseverbesserung bescheren.

Voraussetzung für adjuvante Therapiestudien sollte aber eine differenzierte Patientenstratifizierung sein. Diese wird sich auf eine frühe molekulare Melanomtypisierung und auf eine wesentlich präzisere Stadieneinteilung stützen, als es sie das aktuell noch gültige System des American Joint Committee on Cancer (AJCC) erlaubt [79]. Insgesamt besteht unserer Meinung nach die realistische Chance, die melanombedingte Mortalität erstmals signifikant zu senken - trotz der weiterhin ungebrochenen Inzidenzzunahme dieses Malignoms. Bedingung dafür ist eine konzertierte Kraftanstrengung vieler Beteiligter, die sich diesem gemeinsamen Ziel von verschiedenen Seiten aus nähern, nicht zu vergessen die Krankenkassen, Forschungsförderungsinstitutionen und andere Geldgeber, die die Finanzierung sichern.

\section{Danksagung}

\section{$\nabla$}

Herrn Prof. Dr. med. Lutz Kretschmer von der Universitätshautklinik Göttingen danken die Autoren für viele Jahre der vertrauensvollen und erfolgreichen wissenschaftlichen Zusammenarbeit auf dem Gebiet des Sentinel-Lymphknotens (siehe z.B.@ Abb.4).

\section{Interessenkonflikt}

\section{$\nabla$}

Hans Starz und Christian Haas leiteten zwei Forschungsprojekte, gefördert von Novartis, und nahmen auf Honorarbasis an mehreren Expertentreffen der Firmen Roche und Bristol-Myers-Squibb teil.

Die anderen Autoren geben an, dass kein Interessenkonflikt besteht.

\section{Abstract}

\section{Changes of the Diagnostic and Therapeutic Standards for Malignant Melanoma in the Last 25 Years. State of the Art and Upcoming Advances \\ $\nabla$}

Our knowledge about malignant melanomas has enormously grown within the last 25 years, resulting in a variety of new diagnostic and therapeutic strategies.

The high and still increasing incidence of skin cancer and a successful pilot phase in Northern Germany (Schleswig-Holstein) have led to a nation-wide screening program for skin cancer in Germany in 2008, paid by the medicare insurances. Its effects are under evaluation now.

The early recognition and elimination of regional lymph node metastases has been optimized by the sentinel node technique. It is regularly applied for melanomas of intermediate and high Breslow thickness. In addition, the micromorphometric classification of sentinel node metastases represents a first-rank prognosticator and the best indicator whether a completion lymph node dissection is needed and whether adjuvant treatment with interferon-alpha might be helpful or not.

For patients in advanced stages of metastasis, more successful systemic therapies compared with dacarbazin have become available. Nearly $50 \%$ of all cutaneous melanomas have an activating
V600 mutation in the BRAF kinase, which is part of the MAPK pathway. Vemurafenib and Dabrafenib are orally given drugs, which block the BRAF activity in case of V600 mutation. They usually achieve a quick regression of metastases in the respective patients, and this effect can be stabilized and prolonged by combining one of the BRAF inhibitors with a MEK inhibitor, e.g. Trametinib. Among the immunotherapies, antibodies against downregulating checkpoint receptors on T cells (CTLA4 and PD-1) have shown the best results. While complete remissions of the metastases were rare, continuing tumor control was achieved in a considerable percentage of the patients. The highest percentage was observed in a trial that combined the PD-1 antibody nivolumab with the CTLA4 antibody ipilimumab.

The rational use of all these innovations and - last not least - the optimized collaboration of multiple disciplines in skin cancer centers open the chance, that the melanoma-related mortality might decrease despite a still growing incidence of melanomas.

For this purpose we must learn to find the best "tailored" therapy for each of the upcoming melanoma subentities and for each of the prognostic categories to be defined.

\section{Literatur}

1 Bachter D, Balda B-R, Vogt $H$ et al. Die „sentinel“ Lymphonodektomie mittels Szintillationsdetektor. Eine neue Strategie in der Behandlung maligner Melanome. Hautarzt 1996; 47: 754-758

2 Cochran AJ, Balda B-R, Starz $H$ et al. The Augsburg Consensus. Techniques of lymphatic mapping, sentinel lymphadenectomy, and completion lymphadenectomy in cutaneous malignancies. Cancer 2000; 89: $236-241$

3 Starz H, Balda B-R, Krämer KU et al. A micromorphometry-based concept for routine classification of sentinel lymph node metastases and its clinical relevance for patients with melanoma. Cancer 2001; 91: $2110-2121$

4 Starz H, Cochran AJ, Balda B-R. Die Sentinel-Lymphonodektomie aus histopathologischer Sicht. Akt Dermatol 2002; 28: 273-278

5 Cochran AJ, Starz H. Pathologic aspects. Part II Sentinel Lymph Node Biopsy for Melanoma In: Cody HSIII. Sentinel Lymph Node Biopsy. London: Martin Dunitz Ltd; 2002: 79-90

6 Balda B-R, Starz H. Malignant melanoma. In: Katsambas AD, Lotti TM, eds. European Handbook of Dermatological Treatments. 2nd edition Berlin, Heidelberg: Springer; 2003: 321 - 329

7 Starz H, Haas CJ, Schulz G-M et al. Tyrosinase RT-PCR as a supplement to histology for detecting melanoma and nevus cells in paraffin sections of sentinel lymph nodes. Mod Pathol 2003; 16: 920-929

8 Starz H. Sentinel-Lymphonodektomie. In: Szeimies RM, Hauschild A, Garbe $C$ et al. Tumoren der Haut. Grundlagen, Diagnostik, Therapie. Stuttgart: Thieme; 2010: 345-359

9 Starz H. Ipilimumab for advanced metastatic melanoma. Expert Opin Biol Ther 2012; 12: 981-982

10 Volkenandt $M$, Schmidt $M$, Konz $B$ et al. Klinisch epidemiologische Daten von Patienten mit malignen Melanomen aus dem Bereich des Tumorzentrums München von 1977 bis 1997. Hautarzt 1999; 50: $233-242$

11 Garbe C, Leiter U. Melanoma epidemiology and trends. Clin Dermatol 2009; 27: 3-9

12 Greinert R, Volkmer B, Wende A et al. Prävention von Hautkrebs. Notwendigkeit, Durchführung und Erfolg. Hautarzt 2003; 54: 1152 - 1163

13 Balch CM, Soong S, Ross MI et al. Long-term results of a multi-institutional randomized trial comparing prognostic factors and surgical results for intermediate thickness melanomas (1.0 to $4.0 \mathrm{~mm})$. Intergroup Melanoma Surgical Trial. Ann Surg Oncol 2000; 7: 87-97

14 Cascinelli N, Santinami M, Maurichi A et al. World Health Organization experience in the treatment of melanoma. Surg Clin North Am 2003; 83: $405-416$

15 Balda B-R, Herrmann R. Neoplasien der Haut. In: Huhn D, Herrmann R, Hrsg. Medikamentöse Therapie maligner Erkrankungen. 3. Auflage Stuttgart: Fischer; 1995: 393-405 
16 Khan KH, Goody RB, Harneed $H$ et al. Metastatic melanoma: a regional review and future directions. Tumori 2012; 98: 575-580

17 Ugurel S, Paschen A, Becker JC. Dacarbazine in melanoma: from a chemotherapeutic drug to an immunomodulating agent. J Invest Dermatol 2013; 133: 289-292

18 Argenziano G, Giacomel J, Zalaudek I et al. A clinico-dermoscopic approach for skin cancer screening: recommendations involving a survey of the International Dermoscopy Society. Dermatol Clin 2013; 31: $525-534$

19 Choudhury K, Volkmer B, Greinert $R$ et al. Effectiveness of skin cancer screening programmes. Brit J Dermatol 2012; 167: 94-98

20 Waldmann A, Nolte $S$, Weinstock MA et al. Skin cancer screening participation and impact on melanoma incidence in Germany - an observational study on incidence trends in regions with and without population-based screening. Br J Dermatol 2012; 106: 970 - 974

21 Breitbart EW, Waldmann A, Nolte $S$ et al. Systematic skin cancer screening in Northern Germany. J Am Acad Dermatol 2012; 60: 201 - 211

22 Brod C, Schippert W, Breuninger H. Dysplastic nevus syndrome with development of multiple melanomas. A surgical concept for prophylaxis. JDDG 2009; 7: 773-775

23 Zalaudek I, Kittler H, Blum A et al. Who benefits from prophylactic surgical removal of "dysplastic" nevi? JDDG 2010; 8: 279-280

24 Pellacani G, Cesinaro AM, Seidenari S. Reflectance-mode confocal microscopy of pigmented skin lesions - improvement in melanoma diagnostic specificity. J Am Acad Dermatol 2005; 53: 979-985

25 Pellacani $G$, Farnetani $F$, Gonzalez $S$ et al. In vivo confocal microscopy for detection and grading of dysplastic nevi: a pilot study. J Am Acad Dermatol 2012; 66: e109-121

26 Virchow R. Dritte Vorlesung. Allgemeine Physiologie der Geschwülste. In: Virchow R. Die krankhaften Geschwülste. Berlin: Hirschwald; 1863: $33-56$

27 Morton DL, Wen DR, Wong JH et al. Technical details of intraoperative lymphatic mapping for early stage melanoma. Arch Surg 1992; 127 : $392-399$

28 Giuliano AE, Dale PS, Turner RR et al. Improved axillary staging of breast cancer with sentinel lymphadenectomy. Ann Surg 1995; 222: $394-$ 399

29 Kitagawa $Y$, Fujii H, Kumai $\mathrm{K}$ et al. Recent advances in sentinel node navigation for gastric cancer: a paradigm shift of surgical management. J Surg Oncol 2005; 90: 147-151

30 Wawroschek F, Vogt H, Weckermann D et al. Radioisotope guided pelvic lymph node dissection for prostate cancer. J Urol 2001; 166: 1715 1719

31 Haas CJ, Wagner T, Wawroschek F et al. Combined application of RTPCR and immunohistochemistry on paraffin embedded sentinel lymph nodes of prostate cancer patients. Pathol Res Pract 2005; 200 : $763-770$

32 Starz H, Balda B-R, Bachter D et al. Secondary lymph node involvement from primary large B-cell lymphoma oft he leg: sentinel lymph nodectomy as a new strategy for staging circumscribed cutaneous lymphomas. Cancer 1999; 85: 199-207

33 Düker I, Starz H, Bachter D et al. Prognostic and therapeutic implications of sentinel lymphonodectomy and S-staging in Merkel cell carcinoma. Dermatology 2001; 202: 225-229

34 Krämer K-U, Starz H, Balda B-R. Primary cutaneous CD30-positive large T-cell lymphoma with secondary lymph node involvement detected by sentinel lymphonodectomy. Acta Derm Venereol 2002; 82: 73 - 74

35 Michl C, Starz H, Bachter D et al. Sentinel lymphonodectomy in nonmelanoma skin malignancies. Br J Dermatol 2003; 149: 763 - 769

36 Morton DL on behalf of the MSLT Study Group. Overview and update of the phase III multicenter selective lymphadenectomy trials (MSLT-I and MSLT-II) in melanoma. Clin Exp Metastasis 2012; 29: 699-706

37 Morton DL, Thompson JF, Cochran AJ et al. Final trial report of sentinelnode biopsy versus nodal observation in melanoma. N Engl J Med 2014; 370: 599-609

38 Starz H, Siedlecki K, Balda B-R. Sentinel lymphonodectomy and S classification. A successful strategy for better predicting and for improving the outcome of melanoma patients. Ann Surg Oncol 2004; 11 (Suppl. 03): $162 \mathrm{~S}-168 \mathrm{~S}$

39 Balch CM, Soong SJ, Gershenwald JE et al. Prognostic factors analysis of 17,600 melanoma patients: validation oft he American Joint Committee on Cancer melanoma staging system. J Clin Oncol 2001; 19: 3622 3634
40 Fink AM, Weihsengruber F, Spangl B et al. S-classification of sentinel lymph nodes predicts the results of complete lymph node dissection. Melanoma Res 2005; 15: 267-271

41 Van Akkooi ACJ, Nowecki ZI, Voit C et al. Sentinel node tumor burden according to the Rotterdam criteria is the most important prognostic factor for survival in melanoma patients. A multicenter study in 388 patients with positive sentinel nodes. Ann Surg 2008; 248: 949 - 955

42 Van der Ploeg IM, Kroon BB, Antonini $N$ et al. Comparison of three micromorphometric pathology classifications of melanoma metastases in the sentinel node. Ann Surg 2009; 250: 301 - 304

43 Veenstra HJ, Brouwer OR, van der Ploeg IM et al. Five-year follow-up of 16 melanoma patients with a Starz I-involved sentinel node in whom completion lymph node dissection was omitted. Melanoma Res 2012; 22: $436-439$

44 Migliano E, Bellei B, Govoni FA et al. SLN melanoma micrometastasis predictivity of nodal status: a long term retrospective study. J Exp Clin Cancer Res 2013; 32: 47

45 Van der Ploeg AP, van Akkooi AC, Verhoef $C$ et al. Completion lymph node dissection after a positive sentinel node: no longer a must? Curr Opin Oncol 2013; 25: 152-159

46 Wang $X$, Heller $R$, VanVoorhis $N$ et al. Detection of submicroscopic lymph node metastases with polymerase chain reaction in patients with malignant melanoma. Ann Surg 1994; 220: 768-774

47 Blaheta HJ, Ellwanger U, Schittek B et al. Examination of regional lymph nodes by sentinel node biopsy and molecular analysis provides new staging facilities in primary cutaneous melanoma. J Invest Dermatol 2000; 114: 637-642

48 Howard JH, Thompson JF, Mozzillo $N$ et al. Metastasectomy for distant metastatic melanoma: analysis of data from the first Multicenter Selective Lymphadenectomy Trial (MSLT-I). Ann Surg Oncol 2012; 19: 2547-2555

49 Menzies AM, Haydu LE, Visintin L et al. Distinguishing clinicopathologic features of patients with V600E and V600K BRAF-mutant metastatic melanoma. Clin Cancer Res 2012; 18: 3242-3249

50 Starz H, Moody J, Welzel J et al. Melanomas of the head-and-neck skin with mutation BRAF V600K or BRAF V600R define a melanoma subtype with particular clinical features. J Clin Oncol 2013; 31: abstr 9098

51 Yaman B, Akalin T, Kandiloglu G. Clinicopathological characteristics and mutation profiling in primary cutaneous melanoma. Am J Dermatopathol 2015; 37: 389-397

52 Sosman JA, Kim KB, Schuchter L et al. Survival in BRAF V600-mutant advanced melanoma treated with vemurafenib. N Engl J Med 2012; 366 : $707-714$

53 Young K, Minchom A, Larkin J. BRIM-1, -2 and -3 trials: improved survival with vemurafenib in metastatic melanoma patients with a BRAF (V600E) mutation. Future Oncol 2012; 8: 499-507

54 Hauschild A, Grob JJ, Demidov LV et al. Dabrafenib in BRAF-mutated metastatic melanoma: a multicenter open-label, phase 3 randomised controlled trial. Lancet 2012; 380: $358-365$

55 Gibney GT, Zager IS. Clinical development of dabrafenib in BRAF mutant melanoma and other malignancies. Expert Opin Drug Metab Toxicol 2013; 9: $893-899$

56 Salama AK, Kim KB. Trametinib (GSK1120212) in the treatment of melanoma. Expert Opin Pharmacother 2013; 14: 619-627

57 Long GV, Stroyakovskiy DD, Gogas $H$ et al. Dabrafenib and trametinib versus dabrafenib and placebo for Val600 BRAF-mutant melanoma: a multicentre, double-blind, phase 3 randomised controlled trial. Lancet 2015; 386: $444-451$

58 Becker JC, Bröcker EB, Schadendorf D et al. Imatinib in melanoma: a selective treatment option based on KIT mutation status? J Clin Oncol 2007; 25: e9

59 Faries $M B$, Morton $D L$. Therapeutic vaccines for melanoma: current status. BioDrugs 2005; 19: $247-260$

60 Eggermont AM, Suciu S, Rutkowski P et al. Adjuvant ganglioside GM2$\mathrm{KLH} / \mathrm{QS}-21$ vaccination versus observation after resection of primary tumor $>1.5 \mathrm{~mm}$ in patients with stage II melanoma: results of the EORTC 18961 randomized phase III trial. J Clin Oncol 2013; 31: $3831-3837$

61 Eggermont AM, Suciu S, Testori A et al. Long-term results of the randomized phase III trial EORTC 18991 of adjuvant therapy with pegylated interferon alfa- $2 b$ versus observation in resected stage III melanoma. J Clin Oncol 2012; 30: 3810-3818

62 Hauschild A, Weichenthal M, Rass $K$ et al. Efficacy of low-dose interferon alpha 2a 18 versus 60 months of treatment in patients with pri- 
mary melanoma of $\geq 1.5 \mathrm{~mm}$ tumor thickness: results of a randomized phase III DeCOG trial. J Clin Oncol 2010; 28: 841 -846

63 Pflugfelder A, Kochs C, Blum A et al. S3-guideline "diagnosis, therapy and follow-up of melanoma" - short version. J Dtsch Dermatol Ges 2013; 11: $563-602$

64 Mocellin S, Pasquali S, Rossi CR et al. Interferon alpha adjuvant therapy in patients with high-risk melanoma: a systematic review and metaanalysis. J Natl Cancer Inst 2010; 102: 493-501

65 Trinh VA, Hwu WJ. Ipilimumab in the treatment of melanoma. Expert Opin Biol Ther 2012; 12: 773-782

66 Wolchok JD, Hodi FS, Weber JS et al. Development of ipilimumab: a novel immunotherapeutic approach fort he treatment of advanced melanoma. Ann N Y Acad Sci 2013; 1291: 1 - 13

67 Eigentler TK, Schlaak M, Hassel JC et al. Effectiveness and tolerability of ipilimumab: experiences from 198 patients included in a namedpatient program in various daily-practice settings and multiple institutions. J Immunother 2014; 37: 374-381

68 Robert C, Soria JC, Eggermont AM. Drug of the year: programmed death-1 receptor/programmed death-1 ligand-1 receptor monoclonal antibodies. Eur J Cancer 2013; 49: 2968 - 2971

69 Wolchok JD, Kluger H, Callahan MK et al. Nivolumab plus ipilimumab in advanced melanoma. N Engl J Med 2013; 369: 122-133

70 Postow MA, Chesney J, Pavlick AC et al. Nivolumab and ipilimumab versus ipilimumab in untreated melanoma. N Engl J Med 2015; 372: 2006-2017

71 Larkin J, Chiarion-Sileni V, Gonzalez R et al. Combined nivolumab and ipilimumab or monotherapy in untreated melanoma. N Engl J Med 2015 ; $373: 23-34$
72 The Cancer Genome Atlas Network. Genomic classification of cutaneous melanoma. Cell 2015; 161: $1681-1696$

73 Leiter $U$, Eigentler T, Garbe $U$. Epidemiology of skin cancer. Adv Exp Med Biol 2014; 810: 120-140

74 Stevenson AD, Mickan S, Mallett $S$ et al. Systematic review of diagnostic accuracy of reflectance confocal microscopy for melanoma diagnosis in patients with clinically equivocal skin lesions. Dermatol Pract Concept 2013; 3: 19-27

75 Gambichler T, Schmid-Wendtner MH, Plura I et al. A multicenter pilot study investigating high-definition optical coherence tomography in the differentiation of cutaneous melanoma and melanocytic naevi. JEADV 2015; 29: $537-541$

76 Malvehy J, Hauschild A, Curiel-Lewandrowski C et al. Clinical performance of the Nevisense system in cutaneous melanoma detection: an international, multicentre, prospective and blinded clinical trial on efficacy and safety. Br J Dermatol 2014; 171: 1099-1107

77 Bastian BC. The molecular pathology of melanoma: An integrated taxonomy of melanocytic neaoplasia. Ann Rev Pathol Mech Dis 2014; 9: $239-271$

78 Eggermont AM, Chiarion-Sileni V, Grob JJ et al. Adjuvant ipilimumab versus placebo after complete resection of high-risk stage III melanoma (EORTC 18071): a randomized, double-blind phase 3 trial. Lancet Oncol 2015; 16: $522-530$

79 Balch CM, Gershenwald JE, Soong SJ et al. Final version of 2009 AJCC melanoma staging and classification. J Clin Oncol 2009; 27: 61996206 